\title{
Die Finanzierung eines Studien-, Lehr- und Forschungsaufenthaltes in Frankreich
}

Delia Küsgen et Christiane Schmeken

\section{OpenEdition}

\section{Journals}

Édition électronique

URL : http://journals.openedition.org/ifha/7919

DOI : 10.4000/ifha.7919

ISSN : 2198-8943

Éditeur

IFRA - Institut franco-allemand (sciences historiques et sociales)

Référence électronique

Delia Küsgen und Christiane Schmeken, « Die Finanzierung eines Studien-, Lehr- und

Forschungsaufenthaltes in Frankreich », Revue de l'IFHA [Online], HS | 2014, Online erschienen am: 01 September 2014, aufgerufen am 20 April 2019. URL : http://journals.openedition.org/ifha/7919; DOI 10.4000/ifha. 7919

Ce document a été généré automatiquement le 20 avril 2019.

(C)IFHA 


\title{
Die Finanzierung eines Studien-, Lehr- und Forschungsaufenthaltes in Frankreich
}

\author{
Delia Küsgen et Christiane Schmeken
}

1 Ein Studien- oder Forschungsaufenthalt im Ausland ist eine fachliche und persönliche Bereicherung, aus der oft lebenslange Verbindungen entstehen. Voraussetzung ist, dass der Aufenthalt gut vorbereitet wird. Dies gilt auch für vermeintlich vertraute Nachbarländer wie Frankreich. In erster Linie kommt es dabei auf die sorgfältige Auswahl der Gastinstitution an. Wichtig ist aber auch eine frühzeitige Auseinandersetzung mit der spezifischen Lehr- und Lernkultur im Zielland. Trotz Bologna-Prozess sind die Unterschiede zwischen Deutschland und Frankreich nach wie vor erheblich ${ }^{1}$.

Pro Jahr finden gut 6.000 Studierende aus Deutschland den Weg an eine französische Hochschule. Fast ebenso viele Franzosen studieren in Deutschland. Neben kurz- oder mittelfristigen Aufenthalten im Nachbarland gewinnt zunehmend das Absolvieren ganzer Studiengänge an Bedeutung. Dazu hat vor allem der Bologna-Prozess beigetragen. Ein Ergebnis dieser europäischen Hochschulreform war, dass das Studium europaweit in zwei Studienzyklen von weitgehend identischer Dauer aufgeteilt wird. Durch die gestufte Studienstruktur bietet sich die sogenannte »vertikale« Mobilität, also das Absolvieren des gesamten Bachelor- und vor allem Masterstudiums im Ausland an. Der neu entstandene „Europäische Hochschulraum« zielt zudem darauf ab, Studienleistungen in Europa vergleichbar zu machen, und hat durch die Einführung des European Credit Transfer System (ECTS) europäischen Austauschprogrammen wie dem Erasmusprogramm zu mehr Popularität verholfen. Auch im Bereich von Forschung und Lehre tritt neben den Gastaufenthalt immer häufiger die dauerhafte wissenschaftliche Karriere in Frankreich. Das französische System ist für die Aufnahme qualifizierter Wissenschaftlerinnen und Wissenschaftler aus dem Ausland vergleichsweise offen, und es gibt einen starken akademischen Mittelbau, der dem akademischen Nachwuchs zu einem relativ frühen Zeitpunkt berufliche Sicherheit bietet. Dadurch ist Frankreich trotz niedrigerer Gehälter gerade für Historikerinnen und Historiker und andere Geisteswissenschaftler ein 
attraktives Zielland. Allerdings ist die Zahl der freien Stellen für den Forschungsnachwuchs in den letzten Jahren eher rückläufig. Sehr gute Aussichten bieten sich dagegen nach wie vor denjenigen, die erfolgreich den CAPES oder die agrégation ablegen und damit den Zugang zum Sekundarschulbereich und häufig auch zur universitären Karriere erhalten.

Im Internet gibt es eine Reihe lesenswerter Darstellungen des französischen Hochschulsystems und der entsprechenden Fördermöglichkeiten². Dieser Beitrag, der den Schwerpunkt auf die Angebote für Historiker legt, versteht sich als Ergänzung der vorhandenen Literatur. Neben den vergleichsweise bekannten Angeboten von deutscher Seite liegt ein besonderer Schwerpunkt auf französischen Stipendienprogrammen sowie Institutionen, die im zwischenstaatlichen Bereich angesiedelt sind. Trotz sorgfältiger Recherche können sich die angegebenen Rahmenbedingungen im Laufe der Zeit verändern. Auf den angegebenen Internetseiten finden Sie Aktualisierungen und Ergänzungen. Auf die Aufzählung der europäischen Fördermöglichkeiten wird aufgrund ihrer Fülle hier bewusst verzichtet ${ }^{3}$.

4 Es gibt eine Vielzahl von Stipendien, welche die Kosten eines Auslandsaufenthaltes ganz oder teilweise decken. Voraussetzung ist es, sich früh $\mathrm{zu}$ informieren und das Bewerbungsdossier gut vorzubereiten. Für die meisten der hier genannten Programme beginnt das Auswahlverfahren viele Monate vor dem gewünschten Beginn des Aufenthaltes. Ein vollständiges Dossier erfordert zahlreiche Dokumente (Originale oder Kopien der Diplome, Empfehlungsschreiben, Sprachtests etc.) sowie ein ausgearbeitetes Studien- oder Forschungsprojekt. Damit das Dossier innerhalb der Bewerbungsfrist eingereicht werden kann, sollte die Vorbereitungszeit nicht unterschätzt werden. Für Studienaufenthalte in Frankreich ist es empfehlenswert, ein Sprachniveau von mindestens B1 des Gemeinsamen Europäischen Referenzrahmens vorzuweisen. Jedoch liegt es im Ermessen der Hochschulen, ob ein Sprachnachweis verlangt wird. Die staatlich anerkannten Sprachtests TCF, DELF und DALF werden von den in Deutschland ansässigen Instituts Français, den Volkshochschulen und an einigen Hochschulen angeboten ${ }^{4}$.

\section{Fördermöglichkeiten aus Deutschland}

Die Förderungsmöglichkeiten deutscher Institutionen für einen Frankreich-Aufenthalt sind vielfältig. Einen guten Überblick gibt die Stipendiendatenbank des DAAD, die je nach Ausbildungsstand die geeigneten Programme nicht nur des DAAD, sondern auch anderer Stipendiengeber ermittelt ${ }^{5}$.

Deutscher Akademischer Austauschdienst (DAAD)

Der DAAD ist eine Einrichtung der deutschen Hochschulen und Studierendenschaften zur Pflege und Förderung ihrer internationalen Beziehungen. Als weltweit größte Stipendienorganisation fördert der DAAD sowohl Auslandsaufenthalte deutscher Studierender, Forscherinnen und Forscher in allen Ländern als auch Studien- und Forschungsaufenthalte von Ausländern in Deutschland.

7 Für Studierende und Graduierte deutscher Hochschulen bietet der DAAD Jahresstipendien an und unterstützt kombinierte Studien- und Praxissemester im Ausland. Bei MasterStudiengängen an den Politik- und Verwaltungshochschulen Sciences Po oder Ecole Nationale d'Administration (ENA) werden zusätzlich die Studiengebühren übernommen. Außerdem werden Kurzstipendien für Praktika im Ausland vergeben. 
Doktoranden und Postdoktoranden können für Forschungsaufenthalte Kurz- oder Jahresstipendien beantragen (maximal sechs bzw. zwölf Monate). Postdoktoranden der Geistes- und Sozialwissenschaften haben zudem die Möglichkeit, sich für ein bis zu sechsmonatiges Forschungsstipendium an der Fondation Maison des Sciences de l'Homme (FMSH) zu bewerben ${ }^{6}$.

9 Für den Bereich der Forschungsförderung ist zudem das Programm PROCOPE des DAAD $\mathrm{zu}$ nennen. Dieses Programm des »Projektbezogenen Personenaustauschs" mit Frankreich richtet sich an Hochschullehrerinnen und Hochschullehrer, Wissenschaftlerinnen und Wissenschaftler sowie an den wissenschaftlichen Nachwuchs. Voraussetzung ist, in binationalen Forschergruppen an einem gemeinsamen Projekt zu arbeiten ${ }^{7}$.

10 Neben Forschungsaufenthalten fördert der DAAD schließlich auch die grenzüberschreitende Lehre. Hier werden Lektorate, Kurzzeitdozenturen und Sprachassistenzstellen gefördert ${ }^{8}$. Für die ca. 50 deutschen Lektorinnen und Lektoren in Frankreich bietet der DAAD neben der Aufstockung des Gehalts eine umfangreiche Beratung und Weiterbildung an, welche sie neben fachlicher Fortbildung bei der Wahrnehmung kulturpolitischer Aufgaben unterstützt. Als durch den DAAD geförderter Sprachassistent in Frankreich (insgesamt 4 Stellen) arbeitet man im Projekt DeutschMobil der Robert Bosch Stiftung. Im Zentrum dieser Arbeit steht die Sprachanimation an Schulen.

Alexander von Humboldt-Stiftung (AvH)

11 Die AvH unterstützt Wissenschaftskooperationen zwischen exzellenten ausländischen und deutschen Forscherinnen und Forschern. Mit Forschungsstipendien und -preisen ermöglicht die AvH hoch qualifizierten ausländischen Wissenschaftlerinnen und Wissenschaftlern Forschungsaufenthalte in Deutschland. Die daraus entstehenden Verbindungen mit Wissenschaftlerinnen und Wissenschaftlern in Deutschland fördern die Netzwerkbildung. In diesem Zusammenhang unterstützt die Stiftung auch Forschungsaufenthalte von deutschen Wissenschaftlern im Ausland.

12 Die Feodor Lynen-Forschungsstipendien richten sich an Nachwuchs- und erfahrene Wissenschaftlerinnen und Wissenschaftler und bieten diesen die Möglichkeit, im Ausland ein Forschungsprojekt als Gast bei einem von weltweit über 25.000 Humboldtianern, den Alumni der AvH, zu verwirklichen. Überdurchschnittliche Postdoktoranden haben im Rahmen dieses Programms die Möglichkeit, ein 6- bis 24-monatiges Forschungsprojekt an einem Institut im Ausland mit einem selbst gewählten wissenschaftlichen Gastgeber durchzuführen. Die Promotion darf nicht länger als vier Jahre zurückliegen. Erfahrene Wissenschaftler können ein Stipendium für einen 6- bis 18-monatigen Forschungsaufenthalt im Ausland erhalten, welches in bis zu drei Aufenthalte innerhalb von drei Jahren aufgeteilt werden kann. Voraussetzungen sind, dass die Promotion des Bewerbers nicht mehr als zwölf Jahre zurückliegt, der Bewerber mindestens als Assistant Professor oder Nachwuchsgruppenleiterin bzw. -leiter tätig ist oder auf andere Weise eine mehrjährige eigenständige wissenschaftliche Tätigkeit vorweisen kann.

Deutsche Forschungsgemeinschaft (DFG)

13 Die Forschungsstipendien der DFG sollen Nachwuchswissenschaftlern aller Fachdisziplinen mit abgeschlossener wissenschaftlicher Ausbildung die Möglichkeit bieten, im Ausland ein umgrenztes Forschungsprojekt zu realisieren oder sich in neue wissenschaftliche Methoden einzuarbeiten. Hohe wissenschaftliche Qualität und 
Originalität des Forschungsvorhabens auf internationalem Niveau werden vorausgesetzt. Das Stipendium läuft in der Regel über zwei Jahre ${ }^{10}$.

Begabtenförderungswerke

In Deutschland gibt es zwölf staatlich finanzierte und bundesweit tätige Begabtenförderungswerke, die besonders engagierte und qualifizierte Studierende materiell und ideell fördern. Es hängt von der jeweiligen Stiftung ab, über welchen Zeitraum und wie intensiv Auslandsaufenthalte gefördert werden.

Die Studienstipendien der Stiftungen betragen maximal $670 €$ im Monat. Sie sind abhängig vom Einkommen der Eltern und orientieren sich an der Berechnung des BAföGs. Ein monatliches Büchergeld in Höhe von aktuell $300 €$ wird unabhängig vom Einkommen der Eltern gezahlt. Gegebenenfalls wird der Beitrag zur Kranken- und Pflegeversicherung übernommen. Auslandsphasen während der Promotion werden mit $1.050 €$ im Monat für die Dauer eines Jahres gefördert. Hinzu kommen eine monatliche Forschungskostenpauschale von $100 €$ sowie zusätzliche Fördermittel zur Abdeckung der auslandsbedingten Mehrkosten. Teilweise ist eine Verlängerung möglich.

In den folgenden Abschnitten werden die politischen bzw. konfessionellen Ausrichtungen der einzelnen Stiftungen skizziert, einige besondere Programme vorgestellt und die Voraussetzungen für den Erhalt einer Förderung präzisiert ${ }^{11}$.

Studienstiftung des deutschen Volkes

Die Studienstiftung des deutschen Volkes ist Deutschlands größtes Begabtenförderungswerk. Es ist politisch, konfessionell und weltanschaulich unabhängig. Die Aufnahme in die Studienstiftung erfolgt über Vorschlag von Schule und/oder Hochschullehrerinnen und -lehrern; seit einigen Jahren ist auch eine Eigenbewerbung möglich. Verschiedene Programme unterstützen Studierende bei ihrem Auslandsaufenthalt und erleichtern Absolventen den Einstieg in den Beruf.

Das Carlo-Schmid-Programm, das die Studienstiftung gemeinsam mit dem DAAD durchführt, bietet Absolventen aller Fachrichtungen die Möglichkeit, Praktika bei internationalen Organisationen und EU-Institutionen zu absolvieren. Mit dem HanielStipendienprogramm wird ein Aufbaustudium sowie ein anschließendes Praktikum im Ausland ermöglicht. Es richtet sich an Absolventen aller Fächer ab dem Bachelor mit Interesse für wirtschaftliche Zusammenhänge. Internationale Stipendien für Doktoranden, die zur Geschichte und Kultur des deutschsprachigen Judentums forschen, stehen im Rahmen des Leo Baeck Fellowship Programms zur Verfügung. Regionale Sonderprogramme werden für Aufenthalte in China, Japan, Osteuropa und den USA angeboten ${ }^{12}$.

Politische Stiftungen

19 In Deutschland gibt es sechs Stiftungen, die jeweils einer der etablierten Parteien nahestehen und Studierende und Doktoranden finanziell und ideell fördern und auch Auslandsaufenthalte ihrer Stipendiaten unterstützen:

- Konrad-Adenauer-Stiftung (CDU-nah ${ }^{13}$ ),

- Hanns-Seidel-Stiftung (CSU-nah ${ }^{14}$ ),

- Friedrich-Ebert-Stiftung (SPD-nah ${ }^{15}$ ),

- Friedrich-Naumann-Stiftung für die Freiheit (FDP-nah ${ }^{16}$ ),

- Heinrich-Böll-Stiftung (Bündnis 90/Die Grünen-nah ${ }^{17}$ ),

- Rosa-Luxemburg-Stiftung (DIE LINKE-nah ${ }^{18}$ ), $\underline{\text { Religiöse Förderungswerke }}$ 
Das Cusanuswerk e.V. - Bischöfliche Studienförderung ${ }^{19}$ und das Evangelische Studienwerk e.V. Villigst ${ }^{20}$ sind Einrichtungen der beiden großen deutschen Kirchen. Sie fördern Studenten und Promovierende der katholischen bzw. evangelischen Kirche ideell und finanziell. Das Ernst Ludwig Ehrlich Studienwerk richtet sich an jüdische Studierende und Promovierende. Alle drei Werke setzen neben herausragenden Leistungen auch gesellschaftliches Engagement ihrer Stipendiaten voraus.

\section{Hans-Böckler-Stiftung}

Das Mitbestimmungs-, Forschungs- und Studienförderungswerk des Deutschen Gewerkschaftsbundes (DGB) fördert vorrangig Arbeitnehmerkinder und Absolventen des zweiten Bildungsweges. Voraussetzungen sind neben persönlichen und fachlichen Qualifikationen gewerkschaftliches und gesellschaftspolitisches Engagement ${ }^{21}$.

Stiftung der Deutschen Wirtschaft - Studienförderwerk Klaus Murmann

Stipendiaten des Studienförderwerks Klaus Murmann haben die Möglichkeit, während ihrer regulären Förderzeit für Studien- oder Praxissemester ins Ausland zu gehen und für diesen Zeitraum eine zusätzliche finanzielle Auslandsförderung zu erhalten. Sehr gute Leistungen und Zielstrebigkeit sowie gesellschaftliches Engagement und soziale Kompetenz werden vorausgesetz $\mathrm{t}^{22}$.

Weitere Stiftungen

Es gibt einige weitere Stiftungen, die je nach Forschungsthema junge Wissenschaftler fördern:

\section{Stiftung Robert Schuman}

24 Für Doktoranden, die an einem Thema zum Aufbau Europas oder der europäischen Zusammenarbeit arbeiten. Sehr gute Französischkenntnisse werden vorausgesetzt ${ }^{23}$.

Gerda Henkel Stiftung

25 Sie fördert die historischen Geisteswissenschaften. Entscheidungskriterien für das Stipendium sind Dauer und Verlauf des Studiums, die Abschlussnote, das Lebensalter sowie besondere Qualifikationen. Die Förderdauer beträgt zwei Jahre ${ }^{24}$.

\section{Fritz Thyssen Stiftung}

26 Fördert die Wissenschaft an Hochschulen und gemeinnützigen Forschungseinrichtungen mit dem Ziel der internationalen wissenschaftlichen Zusammenarbeit. Die Forschungsstipendien richten sich an jüngere, promovierte Nachwuchswissenschaftler, die ein auf eigener Initiative beruhendes Forschungsvorhaben verfolgen. Der Grundbetrag der Förderung beläuft sich auf $1.700 €$ monatlich sowie Nebenleistungen zur Deckung der Sach- und Nebenkosten ${ }^{25}$.

\section{Fördermöglichkeiten von französischer Seite}

Deutsche Studierende, die ihr gesamtes Studium in Frankreich absolvieren, haben Anspruch auf die gleichen Beihilfen und Zuschüsse wie französische Studierende. Voraussetzung ist in der Regel ein mindestens einjähriger Aufenthalt in Frankreich bei Förderbeginn sowie Bedürftigkeit ${ }^{26}$.

Wohngeld: Aide personnalisée au logement (APL)

Alle Studierenden in Frankreich, d. h. auch die deutschen Studierenden, können unabhängig von der finanziellen Situation der Eltern einen Antrag auf Wohngeld stellen. 
Die Höhe des Wohngeldes hängt vom persönlichen Einkommen des Vorjahres ab. Ein Antrag (Demande d'aide au logement) kann bei der französischen Familienkasse (Caisse d'Allocation Familiale) gestellt werden ${ }^{27}$.

Arbeitsverträge für Doktoranden: Contrat doctoral

Promotionswilligen in Frankreich wird seit 2009 ein befristeter öffentlich-rechtlicher Arbeitsvertrag gewährt. Diese Form der Promotionsfinanzierung nimmt ständig an Verbreitung zu. Die Dauer des Vertrages beträgt drei Jahre (Verlängerung um ein Jahr möglich) und setzt ein monatliches Gehalt fest (mindestens 1.684,93€) ${ }^{28}$.

Förderungen ausschließlich für internationale Studierende und Wissenschaftler

Die französische Regierung bietet ausländischen Studierenden sowie Wissenschaftlerinnen und Wissenschaftlern eine Reihe von Förderungen an. Die Auswahl erfolgt in der Regel über die diplomatischen Vertretungen Frankreichs im Heimatland der Bewerber. Daneben gibt es Programme, die direkt über das französische Außenministerium (Ministère des affaires étrangères) verwaltet werden. Schließlich ist die Agentur Campus France mit der Abwicklung einiger Stipendienprogramme betraut ${ }^{29}$.

Bourses d'excellence Eiffel

Dieses Programm richtet sich an ausländische Studierende, die später nicht in der Forschung tätig sein möchten. Die Förderung erfolgt für den Zeitraum bis zum Erwerb eines Masters bzw. Aufenthalte bis zu zehn Monaten im Rahmen einer Betreuungskooperation für Doktoranden. Die Bewerber werden ausschließlich von den französischen Hochschulen vorgeschlagen. Bei Zusage für dieses Stipendium verpflichtet sich die Einrichtung, den Bewerber im gewählten Studiengang einzuschreiben.

Bourses d'excellence Major

Dieses Stipendium der Agence pour l'Enseignement Français à l'Étranger und des Ministère des affaires étrangères dient der Förderung hervorragender ausländischer Abiturienten der Lycées français (französische Gymnasien) im Ausland. Es wird ein Stipendium für die Dauer von fünf Jahren bis zum Erwerb des Masters gewährt. Die Bewerbung erfolgt über den jeweiligen Schulleiter.

Jahresstipendien der französischen Regierung

Die französische Regierung bietet - zusätzlich zu den Jahresstipendien des DAAD - eine begrenzte Anzahl von Stipendien für deutsche Studierende an. Sie sind zum Studium an einer staatlich anerkannten Hochschule in Frankreich bestimmt. Diese Stipendien werden durch das gleiche Auswahlverfahren und unter denselben Bedingungen vergeben wie die Jahresstipendien des DAAD ${ }^{30}$.

Französische Gebietskörperschaften (Conseils régionaux)

Bestimmte Conseils régionaux bzw. andere Gebietskörperschaften vergeben Stipendien an ausländische Studierende, die einen Studienaufenthalt in Frankreich planen. Für weitere Informationen können Sie sich an den Conseil régional Ihres zukünftigen Wohnorts in Frankreich wenden.

Einzelne Hochschulen und Forschungsinstitute

Hier sind insbesondere die Stipendien der École Normale Supérieure zu nennen. Bewerber bis zu 26 Jahren mit hervorragenden Studienleistungen können sich nach Abschluss des 1. Masterjahres bewerben ${ }^{31}$. 

Instituts d'études avancées (IEA) sind innovative Einrichtungen, die international hervorragende Senior- und Junior-Wissenschaftlerinnen und -Wissenschaftler aus verschiedenen Disziplinen aufnehmen. Jedes IEA bietet Forschungsaufenthalte von bis zu einem akademischen Jahr an. Wohnung und Arbeitsplatz werden gestellt. Neben $f$ inanzieller und organisatorischer Unterstützung besteht die Möglichkeit, an Seminaren, Kolloquien und Konferenzen teilzunehmen. Weitere Informationen erteilen die einzelnen Institute: Collegium de Lyon, IMéRA de Marseille, IEA de Nantes und IEA-Paris ${ }^{32}$.

Französische Stiftungen

ftungswesen ist in Frankreich im Bereich der Studienförderung im Vergleich zu Deutschland nicht sehr entwickelt. Dennoch lohnt es sich, nach Stiftungen Ausschau zu halten, die den eigenen Forschungsinteressen entgegenkommen. Die im Folgenden genannten Stiftungen sind für Historikerinnen und Historiker von besonderem Interesse: Fondation Napoléon

Die Stiftung fördert Doktoranden, die zum ersten und zweiten französischen Kaiserreich forschen. Jährlich werden sieben Stipendien für Doktoranden in Höhe von $7.500 €$ vergeben ${ }^{33}$.

Fondation pour la Mémoire de la Shoah

Die Stipendien zur Förderung der Forschung zur Geschichte des Antisemitismus, der Shoah und des Judentums richten sich an Doktoranden, insbesondere Doktoranden eines gemeinsam betreuten Promotionsvorhabens (cotutelle). Ebenso werden Kolloquien und Seminare gefördert ${ }^{34}$.

Fondation Hippocrène

Die Stiftung fördert Projekte mit Europabezug im kulturellen, pädagogischen, humanitären oder sozialen Bereich. Diese müssen von gemeinnützigen Vereinen und Einrichtungen getragen werden ${ }^{35}$.

\section{Deutsche Institutionen in Frankreich und deutsch- französische Fördermöglichkeiten}

Deutsches Historisches Institut Paris (DHIP)

Das DHIP - eines der deutschen geisteswissenschaftlichen Institute im Ausland unterstützt Doktoranden im Rahmen thematisch festgelegter Forschergruppen mit Langzeitstipendien bis hin zur Vollpromotion. Die Abwicklung erfolgt analog der französischen contrats doctoraux (s. o.) im Rahmen einer Kooperation mit der École des hautes études en sciences sociales (EHESS). Förderhöhe und Rechtsstatus der Stipendiaten richten sich nach dem contrat doctoral. Eine Einschreibung an der französischen Hochschule ist notwendig.

Kurzzeitmobilitätsstipendien für maximal drei Monate werden für Forschungsvorhaben, die aufgrund der Quellen- bzw. Literaturlage einen Aufenthalt in Frankreich erfordern, vergeben. Die Förderung beträgt $1.500 €$ für Doktoranden und $2.000 €$ für Postdoktoranden. Das Thema sollte im Rahmen des Arbeitsprogramms des Instituts liegen ${ }^{36}$. 

Umgebung planen.

Wissenschaftlerinnen und Wissenschaftler der verschiedenen Karrierestufen können im Rahmen von Resident-Fellow-Einladungen in das DHIP eingeladen werden. Forschungs-, Redaktions- sowie Vortragsaufenthalte werden ebenso gefördert. Die Forschungsinfrastruktur des Instituts, wie z. B. die Bibliothek, kann während des Aufenthalts genutzt werden. Es existieren drei verschiedene Förderlinien, die kurz- und längerfristige Aufenthalte sowie Teil- und Vollstipendien einschließen ${ }^{37}$.

Mit dem Karl-Ferdinand-Werner-Fellowship-Programm werden ein- bis vierwöchige Forschungsaufenthalte in Pariser Archiven und Bibliotheken gefördert. Es richtet sich an historisch arbeitende Wissenschaftlerinnen und Wissenschaftler, die an deutschen Hochschulen in die Lehre eingebunden sind und einen Forschungsaufenthalt in Paris und

Zudem haben Studierende die Möglichkeit, ein Praktikum im DHIP zu absolvieren. Studierende höherer Semester, deren Studium auf deutsch-französische Beziehungen und französische bzw. westeuropäische Geschichte ausgerichtet ist, können sich bewerben. Ein Praktikum dauert in der Wissenschaft in der Regel zwei Monate, in der Bibliothek für Studierende im Fach Bibliothekswesen bis $\mathrm{zu}$ sechs Monate. Es wird nicht vergütet, jedoch besteht die Möglichkeit, sich beim DAAD um ein Stipendium zu bewerben und im Gebäude des DHIP zu wohnen.

Deutsches Forum für Kunstgeschichte (DFK)

Das DFK, das zweite in Paris angesiedelte deutsche geisteswissenschaftliche Institut im Ausland, vergibt Stipendien für Masterstudierende, Doktoranden und Postdoktoranden. Die Dauer der Stipendien ist abhängig vom Umfang der Forschungsarbeiten im Ausland. Die Höhe der Stipendien ist gestaffelt (Master: $500 €$, Doktoranden $1.700 €$, Postdoktoranden $2.000 €$ ).

47 Für Doktoranden und Postdoktoranden der Kunstgeschichte werden Jahresstipendien angeboten. Forschungsstipendien werden für zumeist längerfristig angelegte Projekte vergeben. In den Projekten soll über die konkreten Forschungsinhalte hinaus vor allem die Auseinandersetzung mit den unterschiedlichen Wissenschaftstraditionen und Methoden gefördert werden.

Im DFK können zwei verschiedene Praktika absolviert werden. Das Praktikum Wissenschaft (sechs Monate) richtet sich an Studierende der Kunstgeschichte und wird mit einem Stipendium und einem einmaligen Reisekostenzuschuss des DAAD gefördert. Das Praktikum Bibliothek (mindestens drei Monate) richtet sich an Studierende der Fachhochschulen für Bibliothekswesen im Hauptstudium. Es wird nicht vergütet ${ }^{38}$. Centre interdisciplinaire d'études et de recherches sur l'Allemagne (CIERA)

Das zu den vom DAAD geförderten Zentren für Deutschlandstudien gehörende CIERA ist ein Netzwerk, das aus zehn herausragenden Hochschulen und Forschungsinstituten mit geistes- und sozialwissenschaftlichem Schwerpunkt besteht. Es bietet begleitende Kurse für Doktoranden und Master-Studierende an. Außerdem bietet es finanzielle Unterstützung für binationale junge Forschergruppen, die sich in regelmäßigen Arbeitstreffen - auch am CIERA - austauschen ${ }^{39}$.

Deutsch-Französische Hochschule (DFH)

Die DFH ist eine zwischenstaatliche Einrichtung, unter deren Dach mehr als 180 Mitglieds- und Partnerhochschulen in Deutschland und Frankreich gemeinsame Studien- 
und Forschungsangebote entwickeln. Ziel ist die Stärkung der deutsch-französischen Zusammenarbeit in Forschung und Lehre.

Im Bereich der Lehre steht die Schaffung, Förderung und Durchführung integrierter deutsch-französischer Studiengänge mit Doppelabschluss im Vordergrund. Während der integrierten Auslandsaufenthalte an der Partnerhochschule unterstützt die DFH die Studierenden mit einer Mobilitätshilfe. Einige Doppelabschlüsse werden auch im Fach Geschichte angeboten ${ }^{40}$.

Im Bereich der Forschung wird besonders die Doktorandenausbildung unterstützt. Im Rahmen des deutsch-französischen cotutelle-Verfahrens wird die Vorbereitung und Verteidigung der Dissertation grenzüberschreitend an zwei Hochschulen unterstützt und durchgeführt. Die Mobilitätskosten der Doktoranden und Betreuer werden übernommen (4.500 €, max. drei Jahre). In deutsch-französischen Hochschulkooperationen, den sogenannten Deutsch-Französischen Doktorandenkollegs, fördert die DFH die strukturierte Doktorandenausbildung mit Mobilitätsbeihilfen von $600 €$ monatlich und Auslandsstipendien für maximal zwei Doktoranden von bis zu $1300 €$ monatlich, max. 18 Monate $^{41}$. Die Netzwerkbildung fördert die DFH im Rahmen von deutsch-französischen Sommerschulen und Forschungsateliers für Nachwuchswissenschaftler ${ }^{42}$.

Deutsch-Französisches Jugendwerk (DFJW)

Das DFJW stärkt den Austausch von Jugendlichen durch finanzielle, pädagogische und sprachliche Unterstützung. Ziel ist es, die Mobilität Studierender und junger Forscher im deutsch-französischen und internationalen Rahmen zu verbessern.

Das DFJW stellt Stipendien zur Durchführung eines Forschungsvorhabens an einer französischen Hochschule zur Verfügung. Um diese Stipendien können sich Studierende aller Fachrichtungen $\mathrm{ab}$ dem fünften Semester bewerben. Die Forschungsaufenthalte werden für eine Dauer von bis zu fünf Monaten mit $300 €$ monatlich zzgl. der Reisekosten gefördert. Eine Unterstützung kann auch für einen maximal dreimonatigen Aufenthalt zur Materialsammlung in Frankreich oder ein Praktikum gewährt werden. Ein Praktikum in Frankreich kann ebenso mit einem monatlichen Stipendium in Höhe von $300 €$ monatlich (max. $900 €$ zuzüglich Reisekosten) gefördert werden. Das Praktikum muss mindestens einen Monat andauern und sollte Bestandteil des Studiengangs bzw. dringend empfohlen sein ${ }^{43}$.

\section{Die wichtigsten Fördermöglichkeiten für Historikerinnen und Historiker nach Frankreich auf einen Blick}

\section{Studierende}

1. Jahresstipendium (DAAD),

2. Stipendium eines der zwölf Begabtenförderwerke,

3. Stipendium und Praktikum (DAAD, DHIP, DFK),

4. Deutsch-Französische Studiengänge (DFH),

5. Forschungsaufenthalte, Aufenthalte zur Materialsammlung, Förderung von Praktika (DFJW).

\section{Doktoranden}

1. Langzeitstipendium bis hin zur Vollpromotion (DHIP), 
2. Forschungsstipendium, Kurz- und Jahresstipendien (DAAD),

3. Stipendium eines der zwölf Begabtenförderwerke,

4. Jahresstipendien (DFK),

5. Promotionsstipendien bis zu zwei Jahren (Gerda Henkel Stiftung),

6. Forschungsaufenthalte, Aufenthalte zur Materialsammlung (DFJW),

7. Stipendien für Doktoranden (Fondation Napoléon),

8. Stipendien für Doktoranden (Fondation pour la Mémoire de la Shoah),

9. Doktorandentage, Workshops, Sommerschulen (CIERA),

10. Doktorandenkollegs, cotutelle-Verfahren (DFH).

\section{Postdoktoranden}

1. Forschungsstipendium, Kurz- und Jahresstipendien (DAAD),

2. Feodor-Lynen- Forschungsstipendium (AvH),

3. Forschungsstipendien (DFG),

4. Forschungsaufenthalte an deutschen geisteswissenschaftlichen Instituten (DHIP, DFK),

5. Forschungsaufenthalte an französischen Institutionen (FMSH, IEA).

\section{NOTES}

1. Béatrice Durand, Stefanie Neubert, Dorothee Röseberg, Virginie Viallon, Studieren in Frankreich und Deutschland. Akademische Lehr- und Lernkulturen im Vergleich, Berlin 2006 sowie die Artikel von Pierre Monnet (http://ifha.revues.org/7846), Ulrike Krampl (http:// ifha.revues.org/7851) sowie Falk Bretschneider und Christophe Duhamelle (http:// ifha.revues.org/7868) in diesem Wegweiser. Alle Links wurden am 09.07.2014 überprüft.

2. Hier drei Beispiele: http://www.studis-online.de/Studieren/Auslandsstudium/frankreich.php; http://studieren.de/studieren-in-frankreich.0.html; http://www.allemagne.campusfrance.org/.

3. ${ }^{3}$ Informationen gibt es z. B. auf der Website des DAAD unter http://eu.daad.de/de.

4. Instituts Français, http://www.institutfrancais.de.

5. DAAD, https://www.daad.de/ausland/studieren/stipendium/de/70-stipendien-finden-undbewerben/.

6. Maison des Sciences de l'Homme, http://www.msh-paris.fr/recherche/bourses-de-recherchepost-doctorales/bourses-fernand-braudel-ifer/?taillepolice.

7. DAAD, http://www.daad.de/hochschulen/kooperation/partnerschaft/ppp/05485.de.html.

8. DAAD, https://www.daad.de/ausland/lehren/de.

9. Alexander von Humboldt-Stiftung, http://www.humboldt-foundation.de/web/programmenach-zielgruppen.html.

10. Deutsche Forschungsgemeinschaft, http://www.dfg.de/foerderung/programme/ einzelfoerderung/index.html.

11. Bundesministerium für Bildung und Forschung, http://www.bmbf.de/de/294.php.

12. Studienstiftung des deutschen Volkes, http://www.studienstiftung.de/ stipendienprogramme.html.

13. Konrad-Adenauer-Stiftung, http://www.kas.de/wf/de/42.34. 
14. Hanns-Seidel-Stiftung, http://www.hss.de/stipendium/foerderung/finanziellefoerderung.html.

15. Friedrich-Ebert-Stiftung, http://www.fes.de/studienfoerderung/stipendienprogramme/ vorteil-teamwork.

16. Friedrich-Naumann-Stiftung für die Freiheit, http://www.stipendiumplus.de/deine-werke/ friedrich-naumann-stiftung-fuer-die-freiheit.html.

17. Heinrich-Böll-Stiftung, http://www.boell.de/stipendien/bewerbung/bewerbung-11239.html.

18. Rosa-Luxemburg-Stiftung, http://www.rosalux.de/studienwerk/studienstipendium.html.

19. Cusanuswerk, http://www.cusanuswerk.de/foerderung/stipendien/.

20. Evangelisches Studienwerk Villigst, http://www.evstudienwerk.de/stipendien/studium/ voraussetzung.html.

21. Hans-Böckler-Stiftung, http://www.boeckler.de/20.htm.

22. Stiftung der Deutschen Wirtschaft, http://www.sdw.org/studienfoerderwerk/stipendien/ studienfoerderung.

23. Stiftung Robert Schuman, http://www.robert-schuman.eu/de/.

24. Gerda Henkel Stiftung, http://www.gerda-henkel-stiftung.de/foerderung.

25. Fritz Thyssen Stiftung, http://www.fritz-thyssen-stiftung.de/.

26. Campus France, http://www.allemagne.campusfrance.org/node/55431.

27. Caisse d'Allocation Familiale, http://www.caf.fr.

28. Ministre de l'Enseignement Supérieur et de la Recherche, L'Étudiant, http:// www.etudiant.gouv.fr/pid20439/contrats-doctoraux.html.

29. Ministère des Affaires Étrangères, http://www.diplomatie.gouv.fr/fr/servicesformulaires_831/espace-etudiants_12793/etudier-france_12794/financer-projet-bourses_12805/ index.html.

30. DAAD, https://www.daad.de/ausland/studieren/stipendium/de/70-stipendien-finden-undbewerben/?detailid=390\&fachrichtung=16\&land=26\&status=1\&seite $=1$.

31. École Normale Supérieure, http://www.ens.fr/spip.php?rubrique66\&lang=fr.

32. Réseau français des instituts d'études avancées, http://www.rfiea.fr/candidater/iea.

33. Fondation Napoléon, http://www.napoleon.org/fr/fondation/bourses/index.asp.

34. Fondation pour la Mémoire de la Shoah, http://www.fondationshoah.org/FMS/spip.php? rubrique25\&lang=fr.

35. Fondation Hippocrène, http://www.fondation-hippocrene.fr/index.php?m1=2\&l=fr.

36. Deutsches Historisches Institut Paris, http://www.dhi-paris.fr/de/home/foerderungfellowships/stipendien/stipendienordnung.html?FSize=1.

37. Deutsches Historisches Institut Paris, http://www.dhi-paris.fr/de/home/foerderungfellowships/resident-fellows.html.

38. Deutsches Forum für Kunstgeschichte, http://www.dtforum.org/index.php?id=2.

39. CIERA, http://www.ciera.fr/ciera/. Siehe auch DAAD, http://www.daad-magazin.de/17871/ index.html.

40. Deutsch-Französische Hochschule, http://www.dfh-ufa.org/studium/studienfuehrer/mode/ liste/fr/18/pointer/0.

41. Deutsch-Französische Doktorandenkollegs, www.dfh-ufa.org/forschung/dt-frzdoktorandenkollegs-dfdk.

42. Deutsch-Französische Hochschule, http://www.dfh-ufa.org/forschung.

43. Deutsch-Französisches Jugendwerk, http://www.dfjw.org/studieren. 


\section{RÉSUMÉS}

Der folgende Beitrag gibt einen Überblick über die wichtigsten Finanzierungsmöglichkeiten eines Frankreichaufenthaltes für deutsche Studierende, Doktoranden und Postdoktoranden. Dabei handelt es sich überwiegend um Förderungen, die fachunabhängig vergeben werden. Soweit Fördermöglichkeiten gezielt für Historiker oder Angehörige der entsprechenden Fächergruppen bestehen, werden diese ausführlicher dargestellt. Im ersten Abschnitt werden die Förderorganisationen und Programme auf deutscher Seite vorgestellt, der zweite Teil geht auf die Angebote französischer Institutionen ein. Deutsch-französische Einrichtungen und Programme sind Thema des dritten Abschnitts. Abschließend finden Sie eine Auflistung der jeweils wichtigsten Fördermöglichkeiten für Studierende, Doktoranden und Postdoktoranden.

\section{INDEX}

Schlüsselwörter : Stipendien, Historiker, Auslandsstudium, Praktika, Förderung, Frankreich, Geschichte, Studium, Finanzierung, Forschung, Studienfinanzierung, Mobilität, Auslandsaufenthalt

\section{AUTEURS}

\section{DELIA KÜSGEN}

Delia Küsgen war Praktikantin am DAAD Paris (2012) und ist derzeit DAAD-Jahresstipendiatin an der Universität Paris III - Sorbonne Nouvelle.

Mail: delia_kuesgen[at]web.de

\section{CHRISTIANE SCHMEKEN}

Christiane Schmeken leitet seit 2012 das Pariser Büro des DAAD.

Mail: schmeken[at]daad.de 